\title{
ON THE DUALITY BETWEEN SMOOTHABILITY AND DENTABILITY
}

\author{
TED LEWIS ${ }^{1}$
}

\begin{abstract}
By renorming $l_{1}$ with an equivalent dual norm it is shown that smoothability of the unit ball of a conjugate Banach space $E^{*}$ does not imply dentability of the unit ball of either $E$ or $E^{* *}$. It is also shown that the unit ball may be smoothable yet fail to be smooth at any point.
\end{abstract}

\section{Introduction.}

1.1. A nonempty subset $K$ of a Banach space $E$ is said to be smoothable if for every $\varepsilon>0$ there is an $f$ in $E^{*}$ with $\|f\|=1$ and a closed ball $B \subset E$ such that the following conditions are satisfied:

(i) $\sup f[B]<\sup f[K]$,

(ii) $\{x \in K: f(x) \leqslant \sup f[K]-\varepsilon\} \subset B$.

The notion of smoothability is originally due to Edelstein [2]. The definition given above is as reformulated by Kemp [3].

There is a formal duality between the notion of smoothability and the notion of dentability (see [2] and $\$ 2$ below); and in [2] it was shown that the smoothability and dentability properties of $c_{0}, l_{1}$, and $m$ parallel this formal duality. For example, the unit ball of $l_{1}$ is dentable but not smoothable while the unit ball of $m$ or $c_{0}$ is smoothable but not dentable. In [3] Kemp has generalized this by showing that if the unit ball of any Banach space is dentable then the unit ball of the conjugate space must be smoothable. ${ }^{2}$ At the same time Kemp asked whether smoothability of the unit ball of $E^{*}$ implies dentability of the unit ball of $E$, and whether the unit ball of $E$ is smoothable iff the unit ball of $E^{*}$ is dentable.

In this note we provide an answer to these questions with the following

1.2. THEOREM. There is a conjugate Banach space, $E^{*}$, whose unit ball is smoothable while neither the unit ball of $E$ nor the unit ball of $E^{* *}$ is dentable.

It was also asked in [3] whether smoothability of the unit ball of $E$ implies that the norm of $E$ is Gâteaux differentiable at some point. We shall show

Received by the editors July 26, 1976 and, in revised form, September 28, 1976.

AMS (MOS) subject classifications (1970). Primary 46B99.

Key words and phrases. Smoothability, dentability, Gâteaux differentiability.

${ }^{1}$ Supported in part by NRC Grant A-8761.

${ }^{2}$ This in turn has been extended by Sullivan [5], who has shown that if the unit ball of $E$ is dentable then every bounded subset of $E^{*}$ is smoothable. 
1.3. TheOREM. There exists a Banach space $E$ whose unit ball is smoothable and whose norm is not Gâteaux differentiable at any point.

2. Definitions. If $A$ and $B$ are two subsets of a Banach space $E$ we denote by $A+B$ the set $\{a+b: a \in A, b \in B\}$. The unit ball $\{x:\|x\| \leqslant 1\}$ of $E$ will be denoted $U$, so that $x+r U$ is the closed ball of radius $r$ centered at $x$.

2.1. A nonempty subset $K$ of a Banach space $E$ is said to be dentable [4], if for every $\varepsilon>0$ there is some $x$ in $K$ which is not contained in the closed convex hull of $K \backslash(x+\varepsilon U)$.

Although neither dentability nor smoothability of $K$ implies that $K$ is bounded, we are interested only in the situation where $K$ is bounded. In this case Definitions 1.1 and 2.1 can be recast in such a manner that they become formally dual to each other. This can be seen by noting that a bounded set $K$ is smoothable iff given any $\varepsilon>0$, there exist a point $x$ and a closed hyperplane $\pi$ of $E$, and some positive $\rho$ such that the following three statements hold:

$$
\begin{gathered}
\pi \cap K \neq \varnothing, \\
K \backslash(\pi+\varepsilon U) \subset x+\rho U, \\
\operatorname{dist}(\pi, x+\rho U)>0,
\end{gathered}
$$

where $\operatorname{dist}(A, B)=\inf \{\|a-b\|: a \in A, b \in B\}$.

The three statements that are formally dual to the above are obtained by interchanging the symbols $\pi$ and $x$ in (2.2), (2.3), and (2.4). It is not difficult to verify that the bounded set $K$ is dentable iff the three formally dual statements hold.

For the remainder of this note, $\Gamma$ denotes an arbitrary infinite set, $\alpha$ is a fixed element of $\Gamma$, and if $x$ is a point in either $c_{0}(\Gamma), l_{1}(\Gamma)$, or $m(\Gamma), x^{\prime}$ denotes the point whose value at $\gamma \in \Gamma$ is given by

$$
x^{\prime}(\gamma)=\left\{\begin{array}{l}
x(\gamma) \text { if } \gamma \neq \alpha \\
0 \text { if } \gamma=\alpha
\end{array}\right.
$$

On each of $c_{0}(\Gamma), l_{1}(\Gamma)$, and $m(\Gamma)$, the usual norm will be denoted $\|\cdot\|$. On $c_{0}(\Gamma)$ we define a new norm, $\||\cdot|\|$, by specifying that its closed unit ball be $V$, where

$$
V=\mathrm{Cl}(U+W)
$$

and where

$$
U=\left\{x \in c_{0}(\Gamma):\|x\| \leqslant 1\right\}, \quad W=\left\{x \in c_{0}(\Gamma):(x(\alpha))^{2}+\left\|x^{\prime}\right\|^{2} \leqslant 1\right\} .
$$

(By $\operatorname{cl}(U+W)$ we mean the closure of the subset $U+W$. For $U$ and $W$ as above, $\operatorname{cl}(U+W)=U+W$; but this fact is not required in the sequel, and the proof is therefore omitted.)

In $\$ 3$ we will show that if $E=\left(c_{0}(\Gamma),\||\cdot|\|\right)$, then $E, E^{*}$, and $E^{* *}$ have the properties stated in Theorem 1.2. 
3. Proof of Theorem 1.2. To prove Theorem 1.2, we will first identify the spaces $E^{*}$ and $E^{* *}$, and then show that $E, E^{*}$, and $E^{* *}$ have the desired properties.

3.1. Proposition. Let $E=\left(c_{0}(\Gamma), \||\cdot|||\right)$. Then:

(i) $E^{*}$ is isometric to $\left(l_{1}(\Gamma), \||\cdot|||\right)$ where $\||\cdot|||$ is defined on $l_{1}(\Gamma)$ by

$$
\|\mid x\|=\|x\|+\left[(x(\alpha))^{2}+\left\|x^{\prime}\right\|^{2}\right]^{1 / 2} \text {. }
$$

(ii) $E^{* *}$ is isometric to $(m(\Gamma),\||\cdot|\|)$ where $\||\cdot|||$ is the norm on $m(\Gamma)$ whose closed unit ball is $V^{* *}$ where

$$
\begin{gathered}
V^{* *}=U^{* *}+W^{* *}, \quad U^{* *}=\{x \in m(\Gamma):\|x\| \leqslant 1\}, \\
W^{* *}=\left\{x \in m(\Gamma):(x(\alpha))^{2}+\left\|x^{\prime}\right\|^{2} \leqslant 1\right\} .
\end{gathered}
$$

Proof. On each of $c_{0}(\Gamma), l_{1}(\Gamma)$, and $m(\Gamma)$ define an auxiliary norm \|\| $\mid \cdot\|\|$ by

$$
\|\| x\|\|=\left[(x(\alpha))^{2}+\left\|x^{\prime}\right\|^{2}\right]^{1 / 2}
$$

For $x$ in $c_{0}(\Gamma)$ we have $\|x\| \leqslant\|\| x\|\|\|2\| x \|$, so that on $c_{0}(\Gamma)$ the norm ||||$\cdot|| \mid$ is equivalent to $\|\cdot\|$. In particular, this means that the collection of continuous linear functionals on $\left(c_{0}(\Gamma),\|\||\cdot|||\right)$ may be identified as being $l_{1}(\Gamma)$. We now show that $\left(l_{1}(\Gamma),|||| \cdot||||\right)$ is isometric to $\left(c_{0}(\Gamma),|||| \cdot||||\right)^{*}$. For $x$ in $l_{1}(\Gamma)$ we have

$$
\begin{aligned}
\sup \{x(y): & \left.\|\| y\|\| \leqslant 1, y \in c_{0}(\Gamma)\right\} \\
& =\sup \left\{x(\alpha) \cdot y(\alpha)+x^{\prime}\left(y^{\prime}\right):[y(\alpha)]^{2}+\|y\|^{2} \leqslant 1\right\} \\
& =\sup \left\{\sup \left\{\beta x(\alpha)+x^{\prime}\left(y^{\prime}\right): \beta^{2}+\left\|y^{\prime}\right\|^{2} \leqslant 1\right\}:-1 \leqslant \beta \leqslant 1\right\} . \\
& =\sup \left\{\beta x(\alpha)+\left(1-\beta^{2}\right)^{1 / 2}\left\|x^{\prime}\right\|:-1 \leqslant \beta \leqslant 1\right\} \\
& =\left[(x(\alpha))^{2}+\left\|x^{\prime}\right\|^{2}\right]^{1 / 2} .
\end{aligned}
$$

Thus, as claimed, $\left(l_{1}(\Gamma),\|\| \cdot||||\right)$ is isometric to $\left(c_{0}(\Gamma), \||| \cdot||||\right)^{*}$, and in a similar manner, $(m(\Gamma),|||\cdot||| \mid)$ may be shown to be isometric to

$$
\left(l_{1}(\Gamma), \||| \cdot||||\right) *
$$

The proof of assertion (i) now follows readily. Since $U$ and $W$ are, respectively, the closed unit balls of the isomorphic spaces $\left(c_{0}(\Gamma),\|\cdot\|\right)$ and $\left(c_{0}(\Gamma),|||| \cdot||||\right)$, it follows that $\left(c_{0}(\Gamma), \||| \cdot||\right)$ is isomorphic to $\left(c_{0}(\Gamma),\|\cdot\|\right)$. Again, the set of continuous linear functionals on $\left(c_{0}(\Gamma),\|\| \cdot\|\|\right)$ may be identified as being $l_{1}(\Gamma)$. For $x \in l_{1}(\Gamma)$, the continuity and linearity of $x$ yields

$$
\sup x[V]=\sup x[U]+\sup x[W]=\|x\|+\|\| x\|\| \mid,
$$


that is, $\sup x[V]=\||| x \mid\|$, showing that $\left(c_{0}(\Gamma),\|\mid \cdot\|\right)^{*}$ is isometric to $\left(l_{1}(\Gamma)\right.$, |II $\cdot|| \mid)$.

To prove assertion (ii), we first note that the above results imply that $\left(l_{1}(\Gamma)\right.$, \|\|$\cdot \| \mid)$ is isomorphic to $\left(l_{1}(\Gamma),\|\cdot\|\right)$, and so $m(\Gamma)$ is the set of continuous linear functionals on $\left(l_{1}(\Gamma),\|\| \cdot \| \mid\right)$. Let $B \subset m(\Gamma)$ be the closed unit ball corresponding to the norm on $m(\Gamma)$ which is conjugate to the norm $\||\cdot| \mid$ in $l_{1}(\Gamma)$. To show that $B=V^{* *}$, note that since both $B$ and $V^{* *}$ are weak* compact, it suffices to show that $\sup x[B]=\sup x\left[V^{* *}\right]$ for every $x \in l_{1}(\Gamma)$. By definition of $B$ we have $\sup x[B]=\left|\|x \mid\|\right.$, and by definition of $U^{* *}$ and $W^{* *}$ we have

$$
\|\mid x\|\|=\| x\|+\|\|x\| \|=\sup x\left[U^{* *}\right]+\sup x\left[W^{* *}\right]=\sup x\left[U^{* *}+W^{* *}\right],
$$

which completes the proof of the theorem.

3.2. ThEOREM. The unit ball of $\left(l_{1}(\Gamma),\||\cdot \||)\right.$ is smoothable while neither the unit ball of $\left(c_{0}(\Gamma), \||\cdot|||\right)$ nor the unit ball of $(m(\Gamma), \||\cdot|||)$ is dentable.

Proof. Since the unit ball $U$ of $\left(c_{0}(\Gamma),\|\cdot\|\right)$ is not dentable [4], it follows that $U+W$, and thus $V$ is not dentable, where $U, V, W$ are as defined in (2.6). Similarly, since the unit ball $U^{* *}$ of $(m(\Gamma),\|\cdot\|)$ is not dentable [1], it follows that the unit ball $V^{* *}$ of $(m(\Gamma), \||\cdot|||)$ is not dentable.

To prove that the unit ball $V^{*}$ of $\left(l_{1}(\Gamma),\|\| \cdot \| \mid\right)$ is smoothable, let $\varepsilon>0$ be given, let $z$ be the continuous linear functional on $l_{1}(\Gamma)$ whose value at each point $x$ is given by $z(x)=2 x(\alpha)$ and let $p \in l_{1}(\Gamma)$ be the point for which $p(\alpha)=-\frac{1}{2}$ and $p(\gamma)=0$ for $\gamma \neq \alpha$.

The point $p$ is in the unit ball $V^{*}$, and $\sup z\left(V^{*}\right)=z(-p)=1$. To show that $V^{*}$ is smoothable, it suffices to show that there is some closed ball $B=p+\rho V^{*}$ of radius $\rho<2$ which contains $\left\{x \in V^{*}: z(x) \leqslant 1-\varepsilon\right\}$, for then we would have $\sup z[B]=\rho-1<1=\sup z\left[V^{*}\right]$. In other words, it suffices to show that there is some positive $\rho<2$ such that each point $x$ of $\left\{x \in V^{*}:\left|\|x \mid\| \leqslant 1, x(\alpha) \leqslant \frac{1}{2}(1-\varepsilon)\right\}\right.$ is at distance less than $\rho$ from $p$ (where, of course, distance is measured using the norm $\|\cdot\| \|$ ).

Since $\||x|\|=|x(\alpha)|+\left\|x^{\prime}\right\|+\left(|x(\alpha)|^{2}+\left\|x^{\prime}\right\|^{2}\right)^{1 / 2}$, if $\|x\| \mid \leqslant 1$ we find that

$$
\left\|x^{\prime}\right\| \leqslant(1-2|x(\alpha)|) /(2-2|x(\alpha)|) .
$$

The definition of $p$ implies that $(x-p)^{\prime}=x^{\prime}$ for all $x$, and if $\|x\| \| \leqslant 1$ it now follows from (3.4) that $\|\mid x-p\| \| \leqslant f(x(\alpha))$, where

$$
\begin{aligned}
f(x(\alpha))= & \left|x(\alpha)+\frac{1}{2}\right|+\frac{1-2|x(\alpha)|}{2-2|x(\alpha)|} \\
& +\left[\left|x(\alpha)+\frac{1}{2}\right|^{2}+\left[\frac{1-2|x(\alpha)|}{2-2|x(\alpha)|}\right]^{2}\right]^{1 / 2} .
\end{aligned}
$$


Since $\|\mid x\| \leqslant 1$ and $x(\alpha) \leqslant \frac{1}{2}(1-\varepsilon)$ together imply that $-\frac{1}{2} \leqslant x(\alpha)$ $\leqslant \frac{1}{2}(1-\varepsilon)$, the proof of the theorem will be completed once it is shown that $\sup \left\{f(x(\alpha)):-\frac{1}{2} \leqslant x(\alpha) \leqslant \frac{1}{2}(1-\varepsilon)\right\}<2$. Due to the compactness of the interval $\left[-\frac{1}{2}, \frac{1}{2}(1-\varepsilon)\right]$ and the continuity of $f$ on this interval, we need only show that $f(x(\alpha))<2$ for each $x(\alpha)$ in $\left[-\frac{1}{2}, \frac{1}{2}(1-\varepsilon)\right]$.

Both $\left|x(\alpha)+\frac{1}{2}\right|$ and $(1-2|x(\alpha)|) /(2-2|x(\alpha)|)$ are increasing on $-\frac{1}{2} \leqslant$ $x(\alpha) \leqslant 0$, and so $f$ is also increasing on that interval. It follows that $f(x(\alpha))<$ 2 for each $x(\alpha)$ in $\left[-\frac{1}{2}, 0\right]$. To show that $f(x(\alpha))<2$ for $x(\alpha)$ in $(0$, $\left.\frac{1}{2}(1-\varepsilon)\right]$, let $x(\alpha)=\frac{1}{2}(1-\delta)$, where $\varepsilon \leqslant \delta<1$. Then

$$
\begin{aligned}
f(x(\alpha)) & =1-\frac{\delta}{2}+\frac{\delta}{1+\delta}+\left[\left(1-\frac{\delta}{2}\right)^{2}+\left(\frac{\delta}{1+\delta}\right)^{2}\right]^{1 / 2} \\
& =1-\frac{\delta}{2}+\frac{\delta}{1+\delta}+\left[\left(1+\frac{\delta}{2}-\frac{\delta}{1+\delta}\right)^{2}-\frac{\delta^{2}}{1+\delta}\right]^{1 / 2},
\end{aligned}
$$

and since $\delta^{2} /(1+\delta)>0$, it is clear that $f(x(\alpha))<2$ whenever $0<x(\alpha) \leqslant \frac{1}{2}(1-\varepsilon)$. This completes the proof of Theorem 3.2.

Theorem 1.2 now follows from Theorems 3.1 and 3.2, and Theorem 1.3 will follow from

3.3. ThEOREM. If $\Gamma$ is uncountable, the norm $\|\cdot\| \|$ on $l_{1}(\Gamma)$ is nowhere Gâteaux differentiable, that is, the unit ball $V^{*}$ is not smooth at any point.

Proof. Let $x \in V^{*},\||x \||=1$. It is well known that for uncountable $\Gamma$, the usual unit ball, $U^{*}$, of $l_{1}(\Gamma)$ is not smooth at any point. In particular, let $z_{1}$ and $z_{2}$ be two linearly independent vectors in $m(\Gamma)$ such that for $i=1,2$, $\left\|z_{i}\right\|=1=z_{i}(x /\|x\|)$, and let $z_{0} \in m(\Gamma)$ be such that \|\|$z_{0}\|\|=1=$ $z_{0}(x /\|\| x|\||)$. Then $\left(z_{i}+z_{0}\right)(x)=1$, and so $\left\|z_{i}+z_{0}\right\| \geqslant 1$. On the other hand, $z_{i}+z_{0} \in U^{* *}+W^{* *}=V^{*}$ so $\left\|z_{i}+z_{0}\right\| \mid \leqslant 1$, showing that

$$
\left\|\mid z_{i}+z_{0}\right\|=1=\left(z_{i}+z_{0}\right)(x) \text { for } i=1,2,
$$

so that $V^{*}$ is not smooth at $x$.

We comment that Theorem 1.3 is a parallel of Proposition 1 of [1]. It was shown in [1] that $c_{0}\left(=c_{0}(\omega)\right)$ contains a symmetric closed and bounded convex body which is dentable, but which does not contain a single extreme point; i.e., $c_{0}$ can be renormed so that its closed unit ball is dentable but does not contain any exposed points.

ADDED IN REVISION. R. Anantharaman and J. H. M. Whitfield have independently answered the question concerning the duality between smoothability and dentability [6]. The author also expresses his thanks to the referee for his comments and suggestions.

\section{REFERENCES}

1. M. Edelstein, Concerning dentability, Pacific J. Math. 46 (1973), 111-114. MR 48 \#2730.

2. Smoothability versus dentability, Comment. Math. Univ. Carolinae 14 (1973), 127-133. MR 47 \#9243. 
3. D. C. Kemp, A note on smoothability in Banach spaces, Math. Ann. 218 (1975), 211-217.

4. M. A. Rieffel, Dentable subsets of Banach spaces, with applications to a Radon-Nikodym theorem, Functional Analysis (Proc. Conf., Irvine, Calif., 1966), Academic Press, London; Thompson, Washington, D. C., 1967, pp. 71-77. MR 36 \#5668.

5. F. Sullivan, Dentability, smoothability and stronger properties in Banach spaces, (preprint).

6. R. Anantharaman and J. H. M. Whitfield, Smoothability Banach spaces, Notices Amer. Math. Soc. 23 (1976), A-535. Abstract \#737-46-3.

Department of Mathematics, University of Alberta, Edmonton, Alberta t6G 2G1, CanAda 